\title{
Deep Belief Network Approach for Recognition of Cow using Cow Nose Image Pattern
}

\author{
Rotimi-Williams BELLO*, Abdullah Zawawi Hj TALIB and \\ Ahmad Sufril Azlan Bin MOHAMED
}

School of Computer Sciences, Universiti Sains Malaysia, Pulau Pinang, Malaysia

( Corresponding author's e-mail: sirbrw@yahoo.com)

Received: 25 September 2019, Revised: 24 January 2020, Accepted: 23 February 2020

\begin{abstract}
A deep belief network is proposed to learn the discriminatory cow nose image texture features for a robust representation of cows' features and recognition using a cow nose image pattern. Deep belief network is a deep learning model that is graphically based, and it is applied to learn the extracted feature sets of cow nose image pattern for hierarchical representation by using the training details of the training phase of the system proposed. Deep belief network application is useful in animal biometrics to monitor the animals through its recognition and identification techniques. Biometrics application emanated from computer vision and pattern recognition. Its application plays an important role in registering and monitoring animals through its recognition and identification techniques. Because the existing physicalbased feature representation methods and manual visual feature extractions cannot handle animal recognition, the deep belief network technique is proposed using the animal's visual attributes. An experiment performed under a controlled condition of identification indicated that the proposed method outshines the existing methods with approximately $98.99 \%$ accuracy. Four thousand cow nose images from an existing database of 400 individual cows contribute to the community of research, especially in the animal biometrics for identification of individual cow.
\end{abstract}

Keywords: Animal biometrics, Cow nose image, Convolutional neural network, Deep belief network, Identification

\section{Introduction}

All over the world, cows are reared for different purposes. They can be reared for meat production, milk production, leather production, or as farm tools. However, poor monitoring technique is a hazard to cow breeding and rearing, which leads to fraudulent activities such as rustling and swapping. Such activities may sometimes degenerate ownership disputes and low profitability. Most significantly, cows chare monitored for vaccination management, traceability, health information, performance recording, and ownership attachment [1]. Different animal monitoring and identification methodologies such as global positioning system (GPS), virtual fencing, using of tattoo, tags, radio frequency identification (RFID), sensors, photographs, drawings, descriptions, branding (hot and freeze), ear notching, microchip implants, iris activities, and retina scan, and muzzle or nose prints are known animal recognition and identification methods. However, they come with a number of weaknesses [2]. The main problems with some of these methodologies are (1) low image quality, (2) infliction of injury on the body of an animal, (3) low-frequency coverage, (4) loss of tags (5) duplication. Hence, devising a robust means for cow identification in order to mitigate the iterated challenges is a task that involves state-of-the-art deep learning techniques. 
http://wjst.wu.ac.th

According to Wang et al. [3] and Krizhevsky et al. [4], the deep learning framework's different layers enable the modeling and representation of the problematic data variation to recognize the animal. A cow recognition system based on enhanced deep learning approaches and frameworks is proposed to solve the recognition and identification problems known with the traditional identification methods by learning the nose image pattern, giving the feature proper representation, and classifying them jointly for a specific task [5,6]. According to Wang et al. [3], RFID faces a lot of challenges because of its implementation and management of its protocols and chips at different points.

Deep learning, an emerging field under computer vision, is an approach employed recently by researchers to recognize and detect what the animal looks like as a consequence of the interaction of its genotype and the environment. Deep learning is generally accepted as one of the most recent, reliable, and accurate techniques for extracting features and individual animal representation $[7,8]$. The technology behind deep learning enables its framework to perform learning on the extracted sets of biometrics species feature to represent and identify the species [9].

According to Jain et al. [10] and Giot et al. [11], human biometrics is an important security mechanism that uses human fingerprint images and other unique human image features such as traits, characteristics, and identifiers to assign a unique identity to an individual. These human biometrics identifiers must fulfill operational and behavioral characteristics in nature, which include accuracy and uniqueness [12]. To mimic the human biometrics, animal biometrics has adopted the human biometrics traits to the animal identification method for animal tracking, animal disease trajectory, animal health information, animal performance recording, and many other purposes. However, capturing an animal image, especially that of a cow for biometrics use in an unconstrained environment, needs skill and expertise. This process comes with lots of flaws such as low illumination, background patches, noises, and many more due to instability of the animal posture and the environment. This is because when the images are captured, it greatly affects the accuracy and the overall processing time of the image.

Cow nose image, which is investigated for its uniqueness for identification just as the human fingerprint, could be traced back to the 1920s [13]. When cow nose images were being collected using the paper-based or inked method, special skills to control the animal are inconvenient because they produce low quality and a waste of time. Because there is no standard benchmark for capturing the cow nose image, this research work utilized the existing cow nose image database of the ministries of agriculture, forestry, and wildlife in Nigeria as the benchmark for evaluating the proposed cow identification scheme.

This paper addresses the problem of cow recognition based on biometrics features of cow nose image pattern using deep belief network (DBN) architecture for recognition. A DBN is a deep learning model that is graphically based. It is applied to learn extracted feature sets of cow nose image patterns for hierarchical representation by using the training details of the proposed system's training phase. DBN is extremely useful for unsupervised learning and representation of the extracted features of unlabeled cow nose images taken from a large database. The nose image pattern is made up of robust features of texture, which the proposed cow system for recognition recognizes using the frameworks of the deep learningbased recognition. After the cow recognition system has captured the nose pattern image, the captured image is made to undergo pre-processing to remove the background patches, noises, and other discriminatory particles using Gaussian filtering techniques. Deep learning techniques for the identification of the individual cow were then employed.

Based on the similarity between the human fingerprint and cow nose print, and using the robust features of the nose image database, we proposed a novel method for recognizing and identifying cow based on the nose image characteristics using deep learning convolutional neural network (DL-CNN) architecture.

This research work is primarily for the automatic identification of cows to mitigate swapping. Our literature findings revealed no previous works on the use of cow species and the techniques used in this work. The rest of this work is as follows: presented in section 2 is the research related work; materials and methods employed to achieve the research objectives are presented in section 3; while the results and discussion are presented in section 4; section 5 concludes the research. 


\section{Related work}

This section provides the previous efforts made in identifying individual animals using pattern recognition, computer vision, and biometrics method. Due to more and rapid growth experienced in the area of animal biometrics-based recognition systems for tracking, detection, recognition, identification, and monitoring of individual animals, many researchers have developed an interest in the field, and more efforts are still being put into improving it [14-22].

The physical visual characteristics such as nose images possessed by the target species are what the animal biometrics-based recognition system uses to identify an individual animal. There are 2 recognition techniques employed for the identification of the animal. One recognition technique leaves a permanent mark on the animal for identification purposes, while the other recognition technique leaves a temporary mark.

Examples of the recognition technique that leaves a permanent mark are found in the work of Noviyanto and Arymurthy [23] with their drawbacks. Likewise, examples of the recognition technique that leaves a temporary mark on the body of the animal for identification purposes are found in the work of Barron et al. [9] with their drawbacks. As earlier iterated, RFID for animal identification was the most promising classical identification technique employed to identify the animal.

According to Nasirahmadi et al. [24], the feasibility of employing Delaunay triangulation and image processing methods for the change detection in pigs group lying behavior under the farm business conditions and how the changes help in understanding the environmental factors that affect both the pigs' welfare and health conditions should also be investigated.

Furthering their research, Minagawa et al. [25] presented a cow identification framework using nose images. On white A-5 paper were the nose images captured with conspicuous ink, and the performance evaluation of the proposed approach was made by using filtering techniques. The authors employed the binary transformation processes and structural approaches to analyze the nose image. An Equal Error Rate (EER) of 0.419 was reported respectively.

Similar to Minagawa et al. [25], the technique proposed by authors in [26] for recognition of cow using nose image was also used. The experiment carried out by them in order to evaluate the performance of the proposed approach produced 241 False Non-Match Rates (False NMR) over 560 Genuine Acceptance Rate (GAR), and 5197 False Matches (FM) over 12,160 impostors closely matching with the same 0.419 ERR value respectively.

Scale-invariant feature transform (SIFT) descriptor similar to rectangular gradients histogram (R-HOG) [27] approach was used for the cow recognition framework proposed by Awad et al. [28] to localize and to detect the region of interest (ROI) in the nose images for the cow identification in a database of 90 nose images $(6 \times 15=90)$. They employed the application of SIFT keypoint matching based descriptor for the matching of the nose images. RANSAC technique was used with the SIFT in order to mitigate the noises such as outliers points for better identification. Nonetheless, the proposed approach has no cross-validation of experimental results. There was poor identification accuracy from the noises, such as outliers, poor image quality, and blurriness.

Similar to the work of Awad et al. [28] is the work found in Noviyanto and Arymurthy [29], the authors proposed a matching enhancement technique in SIFT descriptor approach for the recognition of cow in 160 nose images database $(4 \times 4 \times 10=160)$ and this performance of the matching enhancement technique was compared to the approach of the original SIFT with equivalent ERR value of 0.0167 .

Ehsani et al. [30] employed image processing and computer vision-based techniques to model visually intelligent agents. Their model takes visual information as input, and the actions of the agent were directly predicted. In this work, they introduced a DECADE, an ego-centric videos dataset from a dog's perspective. How the dog moves and acts were modeled using the data. However, their work was limited to considering only visual data, even though they testify that intelligent agents employ a variety of input modalities such as sound, touch, smell, and so on when interacting with the world. They also limited their work to the modeling of a specific and single dog. Data could be collected from multiple dogs for generalized evaluation across dogs. 
Kumar et al. [31] proposed a framework to ascertain individual cow's identity based on the image of their face. They used the extracted features of the face to evaluate the performance of their proposed system.

Gaber et al. [32] proposed a nose image-based cow recognition approach for local texture features extraction from the nose images using a local binary pattern (LBP) texture algorithm, a local texture descriptor-based technique. These techniques have contributed to the advancement of automated animal recognition and identification with a need for improvement.

\section{Materials and methods}

Presented in this section are the materials and methods employed in achieving the research objectives. This method was motivated by the drawbacks of the classical animal recognition systems and the need to develop an enhanced automated cow recognition method based on the biometrics system that will be robust and non-invasive for identifying cows.

Presently, the available database for cow nose image pattern cannot be employed for the verification evaluation of the reigning state-of-the-art based recognition and classification algorithms. To cater for these issues, the existing database of 4,000 cow nose images captured from 400 breeds (subject) of a cow using a high megapixel camera (30 megapixels) from the ministries of agriculture, forestry, and wildlife, Nigeria was utilized.

One of the main filtration techniques used for this work is made up of the Gaussian filtering technique. Gaussian filter is one of the good general-purpose filters, and it is one of the current standardized approaches for the unsmooth and wave components separation from a primary surface. With minimal phase distortion, both unsmooth and wave surfaces can be acquired from a single filtering procedure. The difference of Gaussian filter can be found in the differences between 2 Gaussian functions [33]. This is calculated by applying 2 Gaussian operators with different values of $\sigma$ to an image, and their differences generated smoothened images. The expression of Gaussian's difference is

$a(b, c)=a_{1}(\mathrm{~b}, \mathrm{c})-a_{2}(\mathrm{~b}, \mathrm{c})$

Where $\mathrm{a}_{1}(\mathrm{~b}, \mathrm{c})$ and $\mathrm{a}_{2}(\mathrm{~b}, \mathrm{c})$ are 2 Gaussian functions given by;

$a_{1}(\mathrm{~b}, \mathrm{c})=\mathrm{e}^{r 2 / 2 \sigma_{1} 2}$

$a_{2}(\mathrm{~b}, \mathrm{c})=\mathrm{e}^{r 2 / 2 \sigma_{2} 2}$

where $\sigma 1>\sigma 2$

From Eqs. (1) - (3);

$a(\mathrm{~b}, \mathrm{c})=\frac{r^{2}}{\mathrm{e}^{2 \sigma_{1} 2}}-\frac{r^{2}}{\mathrm{e}^{2 \sigma_{2}{ }^{2}}}$

Figure 1 shows some samples of cow nose images from the database. Figure 2 shows the database containing blurred nose image patterns; affected by the unconstrained environment and postures of the cow leading to poor quality of the images. Using [16,20,21,31], we filtered the images to get rid of the blurred, background patches and low illumination from the nose images.

As earlier iterated, the existing database of 4000 cow nose images captured from 400 breeds (subject) of cow was utilized for the experiment from which the discriminatory texture features of the nose's beads and the ridges were extracted at the pre-processing stage as shown in Figure 3 (a, b) while Figure 4 shows the colored image of the cow nose with beads and ridges. 

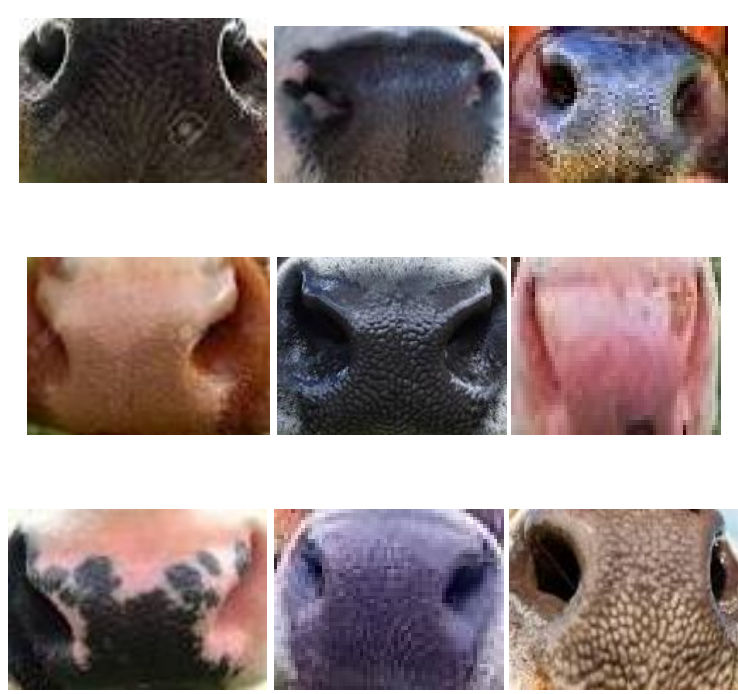

Figure 1 Nose images from database.

One of the discriminatory features of the nose image pattern, beads image features consist of an image pattern of anon-uniform in the cow nose images. The ridges image features are the image patterns that are uniform which is very synonymous to the ridges found on the human fingerprint images.

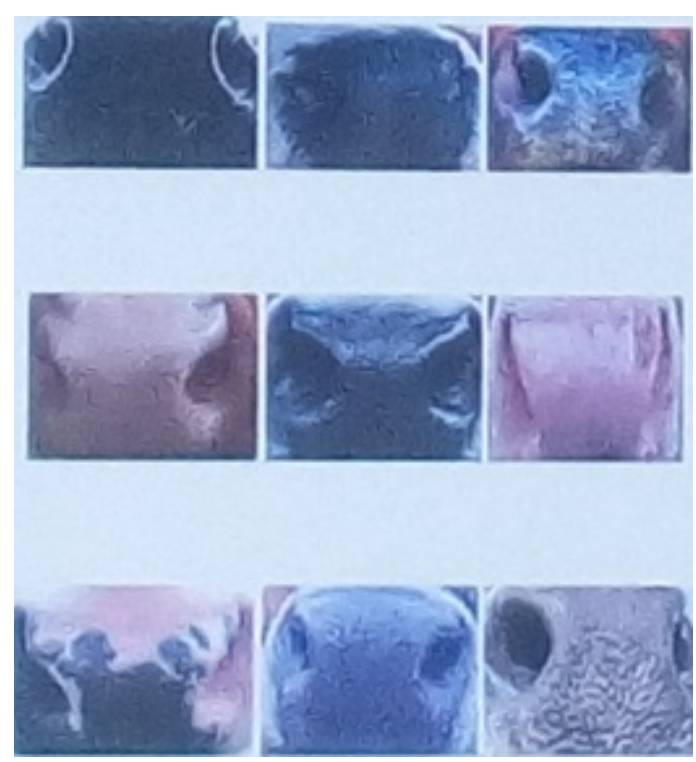

Figure 2 Blurred and poor illumination of nose images. 


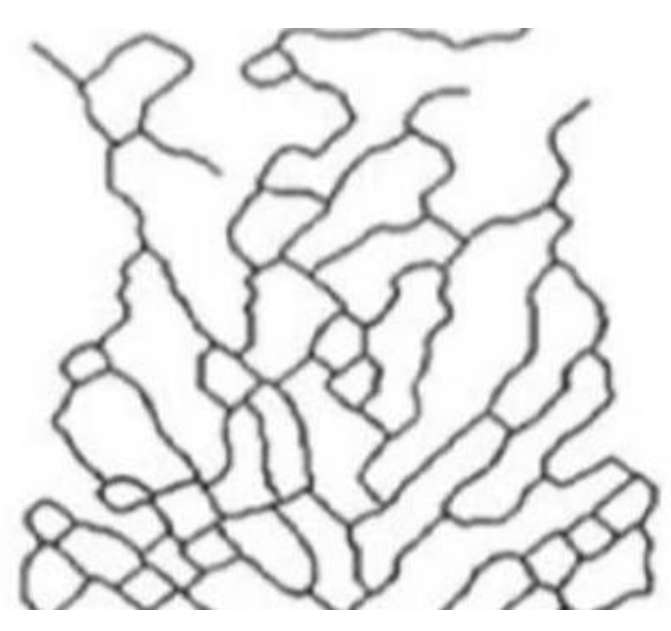

a

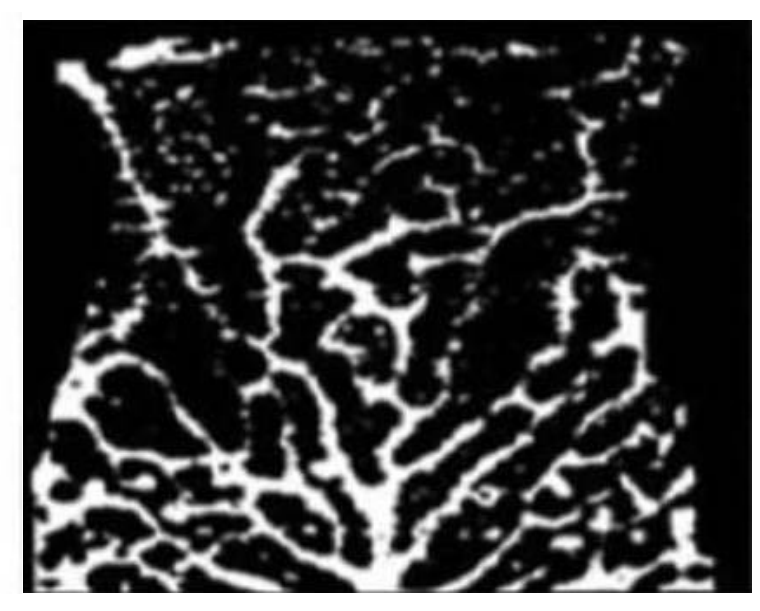

b

Figure 3 (a) Conversion of image to Gaussian image (removal of background) Figure 3 (b) Filtration of discriminatory features

Figure 5 shows the framework of the deep learning that was used for the proposed cow recognition system. One of the main advantages of the difference of Gaussian as a filtering technique (Figure 3 (a)) is the ability to use it for both gray and color images.

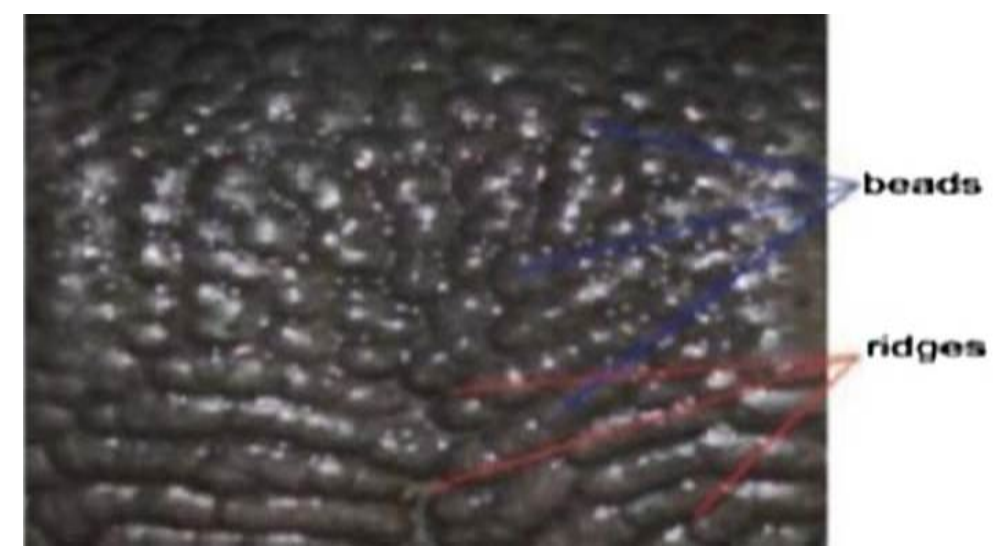

Figure 4 Colored image of cow nose with beads and ridges.

In enhancing the identification process, various image pre-processing techniques were applied in order to remove the patches and reduce the noises from the nose images that were captured. Low illumination and poor image quality are the most 2 fundamental challenges confronting image acquisition, especially cow nose image. The cow nose images captured in an unconstrained environment were converted to grayscale images in order to reduce the patches and the noises captured with them [33]. The 
http://wjst.wu.ac.th

converted images were improved upon by contrast limited adaptive histogram equalization-based image processing technique.

The pre-processing technique accepts the images in their color form and converts them to grayscale before fetching them into the filter for removal of the patches and the noises contained in the captured cow nose images. The feature extraction involves the convolution and pooling operations on the images until the images get to the classifier for classification analysis and the desired output generation. The removal of the noises was carried out using an autoencoding technique. The auto encoding technique is used for the encoding and decoding of the extracted features [34]. Stack denoising autoencoder (SDAE) is a deep learning technique that initializes a deep network. This work is used to encode and decode the texture features of the cow nose image pattern. These texture features were extracted and encoded for the optimum representation of the feature. The 2 primal auto encoder components are the encoder and the decoder. For example, if (A) is an input, an encoder maps it to the hidden layer nodes using a function that is called deterministic mapping function. ( $\mathrm{f}: \mathrm{h}=\mathrm{f}(\mathrm{A})$ ) as shown in Equations 5 and 6 .

$f=G \theta A=s(w \cdot A+\Delta)$

where $\theta=(w, \Delta)$ is the parameter set, s represents the sigmoid, (w) is $\alpha \times \alpha$ weight matrix. $\Delta$ is the offset vector of size $\alpha^{\prime}$. To map the feature vector a' of $\alpha$ dimension, using a decoder function $G^{\prime} \theta$, feature $\mathrm{f}$ is applied such that,

$Y^{\prime \prime}=G^{\prime} \theta^{\prime} f=s\left(w \cdot A^{\prime}+\Delta^{\prime}\right)$

where the decoder parameter $\theta=\left(w^{\prime}, \Delta^{\prime}\right)$ is set.

\section{Deep belief network basics experimental setup}

Problems setting: Given a training set of pre-processed image pattern data

$T=\left\{\left(x_{1} y_{1}\right),\left(x_{2} y_{2}\right), \ldots,\left(x_{N} y_{N}\right)\right\}$

of which $\left(x_{1} y_{1}\right), i=1,2, \ldots, N$ denotes the sample point, $x_{i} \in X \subseteq R^{n}$ is the sample image data while $y_{i} \in Y$ is the corresponding tag of the label; the recognition procedure of proposed system is to input data set $T$ to the DBN network, find the mapping between input $X$ and output $Y$ to form a generative joint probability distribution model formula $P(X, Y)$, generate the output $y_{N+1}$ by

$y_{N+1}=\arg \max _{y_{N+1}} \hat{P}\left(y_{N+1} \mid x_{N+1}\right)$

for a given prediction sample $x_{N+1}$, and judge the image classification of $x_{N+1}$ according to $y_{N+1}$. The system contains the following parts as shown in Figure 5.

The proposed cow nose image pattern identification is composed of a multi-layer Restricted Boltzmann Machine (RBM) as shown in Figure 7 and a back propagation (BP) network layer, wherein the multi-layer RBM will be used to input data feature learning to achieve abstraction and dimensionality reduction of data through the hierarchical feature learning; BP network layer is a categorical network, and it is to categorize the abstracted higher-level features through softmax function. The softmax function, also known as softargmax or normalized exponential function, is a function that takes as input a vector of $\mathrm{K}$ real numbers, and normalizes it into a probability distribution_consisting of $\mathrm{K}$ probabilities proportional to the exponentials of the input numbers.

The first part as shown in Figure $\mathbf{5}$ is "preprocessed cow nose image" which is introduced as input to an optimized DBN technique for feature extraction and classification. 
The second part is "pre-training." For a given training set of image data $=\left\{x_{1}, x_{2}, \ldots, x_{N}\right\}$, the learning system obtains a model through learning (or training) to describe the mapping relationship between input and output variables. This work assumed that RBM model has this descriptive ability, therefore it consists of several layers, through which the input is the image expression data vector while the output is the abstracted higher-level feature vector. Each layer of RBM networks undergoes individually unsupervised training to ensure that feature information is preserved to the uttermost as feature vectors are mapped to different feature spaces. To construct the joint distribution model of visible layer and the hidden layer through energy function, the joint probability maximum likelihood of training sample under model parameter $\widehat{\theta}$ is calculated by;

$P(v \mid \widehat{\theta})=\frac{1}{Z(\theta)} \sum e^{-E(v, \mid \theta)}$

The third part is "fine tuning." Fine-tuning is a common strategy in deep learning to carry out supervised learning through tagged sample training set $T=\left\{\left(x_{1}^{\prime}, y_{1}\right),\left(x_{2}^{\prime}, y_{2}\right), \ldots,\left(x_{N}^{\prime}, y_{N}\right)\right\}$. After that, the top feature vectors corresponding to sample output by the multi RBM network are formed based on the training set of statistical classification structure. This part is a BP network; it will take a specific dimension feature vector to a softmax function. In order to get the best connection weights, this work considered solving the following optimization problem using particle swarm optimization (PSO), so that the loss of function in the training set is minimized.

$\hat{\theta}=\operatorname{argmax}_{\theta} \frac{1}{N} l\left(\theta, x_{n}, t_{n}\right)$

The last part is the "class identification." Tested sample $x_{N+1}$ as network input is subjected to feature learning and abstraction through a network model training to produce a corresponding output $y_{N+1}$ by;

$y_{N+1}=\operatorname{argmax} \hat{P}\left(y_{N+1} \mid x_{N+1}\right)$

and thus achieve classification. 


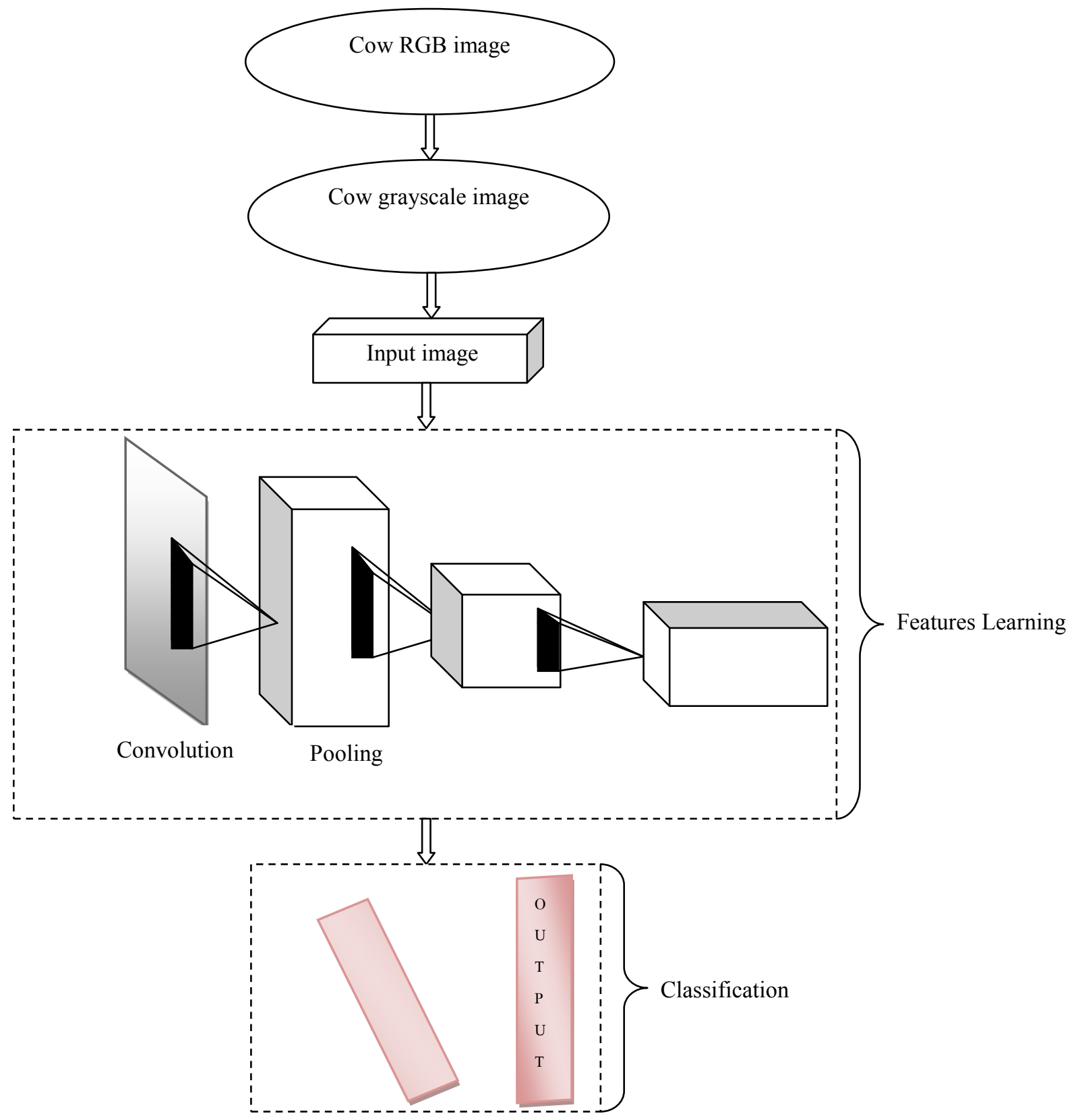

Figure 5 Neural network with convolutional layers for cow recognition.

Technically, CNN is modeled for the training and testing of each input cow nose image which will pass it through a series of convolution layers with filters, pooling, fully connected layers and softmax function for the cow nose image classification with probabilistic values between 0 and 1 . 


\section{Results and discussion}

Having tried out the effectuality of the proposed deep learning approach using the cow's nose image pattern for the recognition and identification of cow, the comparison with other recognition algorithms is attained to evaluate the accuracy of the identification in proliferation settings.

By evaluating the experimental results' performance, the cow nose image database is segmented as follows: (1) the training phase and (2) the testing phase. 100 nose images $(10$ cows (subject) $\times 10$ images of each subject) were used to train the proposed deep learning approach in the training phase. 300 pairs of testing (30 cows (subject) $\times 10$ images of each subject) of nose image pattern in each fold were used for testing the probe images in the testing phase.

For the training of the proposed framework, the deep belief network needs a monolithic database amount. Although, with the number of cow nose images in the database, it is not satisfactory enough to train the network with a database of 4,000 worth of cow nose images. Hence, to fine-tune the weight between the input and the hidden layer and determine the proposed deep learning approach's pre-training, a transfer learning approach was applied.

For the evaluation of the performance, the local feature descriptor technique was used to extract and encode texture features of cow nose image pattern, though, in this paper, handcrafted texture feature descriptor techniques were used to extract the beads and ridges texture feature. As earlier mentioned, the normalization and the descriptor process help mitigate external factors such as low illumination, poor image quality, and background patches affecting the captured images. In performing the tasks involved in this process, cells were converted to blocks. During this process, blocks were overlapped, and cells shared among the blocks and normalized separately. SIFT and R-HOG are similar, though; they do not align to their dominant orientation. To compute R-HOG, $\mathrm{m} \times \mathrm{m}$ grids over $\mathrm{n} \times \mathrm{n}$ pixel cells and histogram bins are needed, where $\mathrm{m}$ is the number of cells in each block.

Figure 6 shows the R-HOG blocks composed of $3 \times 3$ cells of $6 \times 6$ pixels. Nonetheless, the best configuration is to use $2 \times 2$ cells of $8 \times 8$ pixels with 9 histogram bins. 100 nose images equivalent to (10 cows (subject) $\times 10$ images of each subject) were chosen randomly for system training and 300 nose images equivalent to ( 30 cows (subject) $\times 10$ images of each subject) were used for the testing with up to 4 images per subject. The experimental results are reported and analyzed as found in Tables $\mathbf{1}$ and $\mathbf{2}$.

As it is shown in Table1, the performance of the deep belief network framework for identification using DBN provides the highest accuracy in identifying individual cow. Table 1 also illustrates the average accuracy of identification based on individual patches of the nose image pattern to recognize individual cows. The importance of cow nose image pattern is well illustrated in Table 2, where identification accuracy of deep belief network is calculated by considering the different number of nose image features among the ones already calculated using the proposed deep learning framework.

With the increase in usage of the number of texture features of the cow's nose image pattern, the accuracy of the identification is increasing gradually in the 2-deep learning algorithms. The accuracy of identifying the 2-deep learning frameworks increased with every increase in the number of features of selected patches nose image texture features. A deep learning algorithm provided a robust representation of the nose image features in the different layers of the proposed framework after the selection of 400 images of features from each patch $(200 \times 200$ pixels $)$. The selected discriminatory set of the nose image texture features reduce whenever the size of each patch of nose images is reduced.

The logistic regression classifier is for the classification based on the $\mathrm{h}$ (l) (Equations 5 and 6), DBN learning model's last hidden layer. The training of the proposed DBN learning model follows the work of Vincent et al. [35], Bengio [36], and Bengio et al. [37], as illustrated in Figure 7, where the construction of multiple RBM models applies the DBN learning model. Stacked on top of layers, which consists of multiple nodes per layer fed into the next layer, is the RBM classification. RBM's basic working model framework for cow recognition ensures that the DBN deep learning framework serves to extract and learn the cow nose images' texture features extracted sets.

This implies that all the nose image pattern patches have been collectively calculated (as shown in Tables 1 and 2) to describe and represent the discriminatory set of nose image features to the utmost extent than a set of few patches. 
Table $1 \mathrm{CNN}$ and DBN deep learning approaches recognition accuracy.

\begin{tabular}{lc}
\hline Proposed techniques & Recognition accuracy (\%) \\
\hline Convolutional Neural Network & 79.59 \\
Deep Belief Network & 90.95 \\
\hline
\end{tabular}

Table $2 \mathrm{CNN}$ and DBN deep learning approaches recognition accuracy.

\begin{tabular}{ccc}
\hline Number of images & \multicolumn{2}{c}{ Recognition accuracy (\%) } \\
\cline { 2 - 3 } & $\begin{array}{c}\text { Convolutional Neural } \\
\text { Network }\end{array}$ & Deep Belief Network \\
\hline 50 & 61.55 & 62.76 \\
100 & 64.57 & 65.78 \\
150 & 68.87 & 68.98 \\
200 & 72.66 & 72.99 \\
250 & 73.67 & 74.87 \\
300 & 77.89 & 78.86 \\
350 & 79.76 & 79.77 \\
400 & 95.97 & $\mathbf{9 8 . 9 9}$ \\
\hline
\end{tabular}

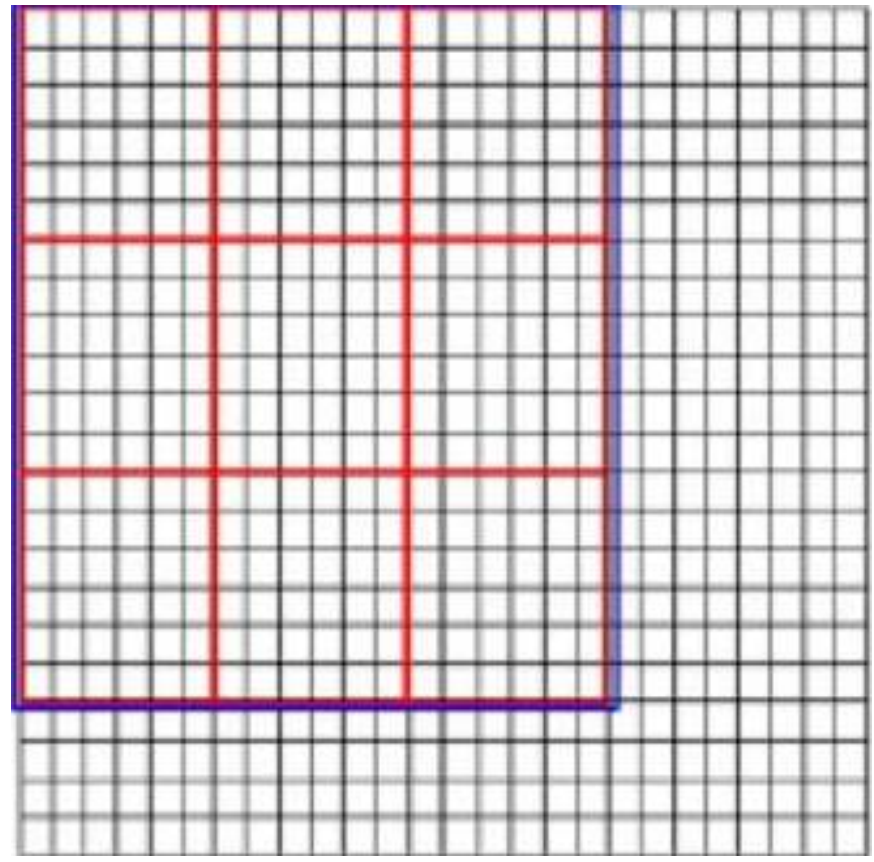

Figure 6 Representation of cells (red), R-HOG (blue), pixels (black). 


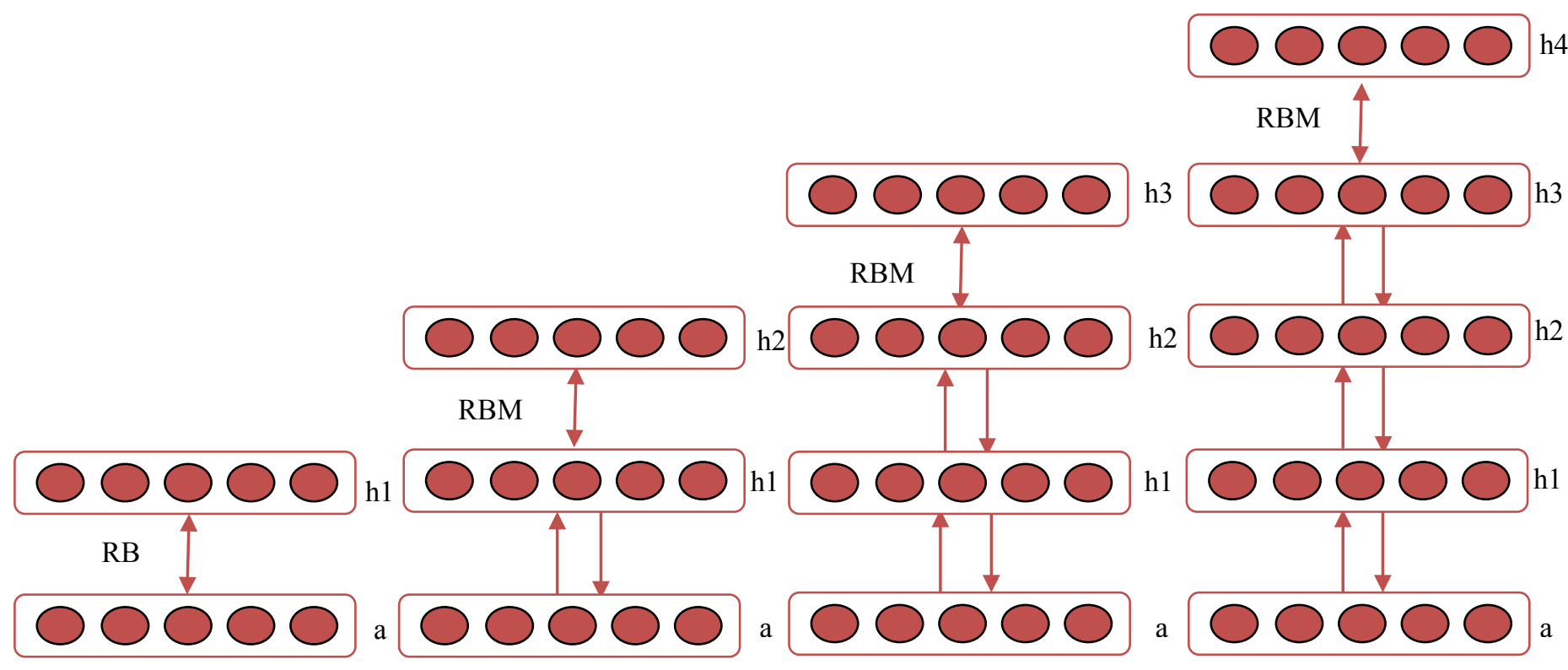

Figure 7 DBN learning model architecture.

\section{Conclusions}

The idea behind human fingerprint image capturing and analysis for human recognition and identification was employed in this work for individual cow recognition and identification. Using the cow nose image texture patterns, the minor points in the human fingerprint patterns are similar to the cow nose print patterns, which are robust and full of rich, dense texture features and contain discriminatory patterns such as bead and ridge patterns.

A deep learning approach was employed to learn the discriminatory features of cow nose images with perfect representation. The research contributed to the advancement of knowledge in the field of computer vision and pattern recognition as follows; 1) the research digs into new enhanced methods for finding the unparalleled means of individual cow identification using the approaches of deep learning; 2) for the encoding and decoding of the extracted prominent nose image pattern texture features of the cow, a deep learning-based stacked denoising autoencoder framework was used; 3) the DBN and CNN deep learning approaches were proposed to learn nose image texture in order to represent the different deep neural network layers with deep learning framework; 4) the approaches used which were based on deep learning framework are suited to address the essential variations of nose image of a cow in the free environment due to background patches, poor image quality, low illumination resulted from the unstable postures of the cow.

The uniqueness and the changeless textural patterns of the beads and the ridges found on the cow nose image serve as biometrics characteristics to identify cow just like a human fingerprint serves as a unique human identification method. Future work includes recognition of animal in real-time using Fuzzy CMeans and Deep Belief Network.

\section{Acknowledgements}

We appreciate the prompt response and assistance rendered to us by the Ministries of Agriculture, Forestry, and Wildlife, Nigeria in retrieving vital information and materials used for this research. 
http://wjst.wu.ac.th

\section{References}

[1] M Vlad, RA Parvulet and MS Vlad. A survey of livestock identification systems. In: Proceedings of the $13^{\text {th }}$ WSEAS International Conference on Automation and Information. 2012, p. 165-70.

[2] CM Roberts. Radio frequency identification (RFID). Comput. Secur. 2006; 25, 18-26.

[3] Z Wang, Z Fu, W Chen and J Hu. A RFID-based traceability system for cattle breeding in china. In: Proceedings of the 2010 IEEE International Conference on Computer Application and System Modeling, Taiyuan, China, 2010, p. V2-567.

[4] A Krizhevsky, I Sutskever and G Hinton. Imagenet classification with deep convolutional neural networks. In: Proceedings of the Advances in Neural Information Processing Systems. Curran and Associates, Red Hook, NY, USA, 2012, p. 1097-105.

[5] C Farabet, C Couprie, L Najman and Y LeCun. Learning hierarchical features for scene labeling. IEEE Trans. Pattern Anal. Mac. Intell. 2013; 35, 1915-29.

[6] Y Sun, X Wang and X Tang. Deep convolutional network cascade for facial point detection. Proc. IEEE Conf. Comput. Vis. Pattern Recognit. 2013; 2013, 3476-83.

[7] S Kumar and SK Singh. Visual animal biometrics: Survey. IET Biometrics 2016; 6, 139-56.

[8] S Kumar, SK Singh, T Datta and HP Gupta. A fast cattle recognition system using smart devices. In: Proceedings of the 2016 ACM conference on Multimedia, Amsterdam, Netherlands, 2016, p. 742-3.

[9] UG Barron, F Butler, K McDonnell and S Ward. The end of the identity crisis? Advances in biometric markers for animal identification. Irish Veterinary J. 2009; 62, 204-8.

[10] AK Jain, AA Ross and K Nandakumar. Introduction to biometrics. Springer Science and Business Media, 2011.

[11] R Giot, M El-Abed and C Rosenberger. Fast computation of the performance evaluation of biometric systems: Application to multibiometrics. Future Generat. Comput. Syst. 2013; 29, 788-99.

[12] AK Jain, A Ross and S Prabhakar. An introduction to biometric recognition. IEEE Trans. Circ. Syst. Video Tech. 2004; 14, 4-20.

[13] WE Petersen. The identification of the bovine by means of nose-prints. J. Dairy Sci. 1922; 5, 24958.

[14] HS Kohl and T Burkhart. Animal biometrics: Quantifying and detecting phenotypic appearance. Trends Ecol. Evol. 2013; 28, 432-41.

[15] S Reiter, G Sattlecker, L Lidauer, F Kickinger, M Öhlschuster, W Auer, V Schweinzer, D KleinJöbstl, M Drillich and M Iwersen. Evaluation of an ear-tag-based accelerometer for monitoring rumination in dairy cows. J. Dairy Sci. 2018; 101, 3398-411.

[16] C Seijas, G Montilla and L Frassato. Identification of Rodent Species Using Deep Learning. Computación y Sistemas. 2019; 23, 257.

[17] MF Hansen, ML Smith, LN Smith, MG Salter, EM Baxter, M Farish and B Grieve. Towards onfarm pig face recognition using convolutional neural networks. Comput. Ind. 2018; 98, 145-52.

[18] S Kumar and SK Singh. Cattle recognition: A new frontier in visual animal biometrics research. In: Proceedings of the National Academy of Sciences. India, 2019, p. 1-20.

[19] MS Norouzzadeh, A Nguyen, M Kosmala, A Swanson, MS Palmer, C Packer and J Clune. Automatically identifying, counting, and describing wild animals in camera-trap images with deep learning. Proc. Natl. Acad. Sci Unit States Am. 2018; 115, E5716-E5725.

[20] TT Zin, CN Phyo, P Tin, H Hama and I Kobayashi. Image technology based cow identification system using deep learning. In: Proceedings of the International Multi Conference of Engineers and Computer Scientists, 2018.

[21] S Kumar, A Pandey, KSR Satwik, S Kumar, SK Singh, AK Singh and A Mohan. Deep learning framework for recognition of cattle using muzzle point image pattern. Measurement 2018; 116, 117.

[22] IA Iswanto and B Li. Visual object tracking based on mean-shift and particle-Kalman filter. Proc. Comput. Sci. 2017; 116, 587-95.

[23] A Noviyanto and AM Arymurthy. Automatic cattle identification based on muzzle photo using 
http://wjst.wu.ac.th

speed-up robust features approach. In: Proceedings of the $3^{\text {rd }}$ European Conference of Computer Science. 2012, p. 114.

[24] A Nasirahmadi, U Richter, O Hensel, S Edwards and B Sturm. Using machine vision for investigation of changes in pig group lying patterns. Comput. Electron. Agr. 2015; 119, 184-90.

[25] H Minagawa, T Fujimura, M Ichiyanagi, K Tanaka and M Fangquan. Identification of beef cattle by analyzing images of their muzzle patterns lifted on paper. In: Proceedings of the $3^{\text {rd }}$ Asian Conference for Information Technology in Asian Agricultural Information Technology and Management. 2002, p. 596-600.

[26] B Barry, U Gonzales-Barron, K McDonnell, F Butler and S Ward. Using muzzle pattern recognition as a biometric approach for cattle identification. Trans. ASABE 2007; 50, 1073-80.

[27] N Dalal and B Triggs. Histograms of oriented gradients for human detection. In: Proceedings of the IEEE Computer Society Conference on Computer Vision and Pattern Recognition. 2005, p. 886-93.

[28] AI Awad, HM Zawbaa, HA Mahmoud, EHHA Nabi, RH Fayed and AE Hassanien. A robust cattle identification scheme using muzzle print images. In: Proceedings of IEEE Federated Conference on Computer Science and Information Systems. 2013, p. 529-34.

[29] A Noviyanto and AM Arymurthy. Beef cattle identification based on muzzle pattern using a matching refinement technique in the sift method. Comp. Electr. Agr. 2013; 99, 77-84.

[30] K Ehsani, H Bagherinezhad, J Redmon, R Mottaghi and A Farhadi. Who let the dogs out? Modeling dog behavior from visual data. In: Proceedings of the IEEE Conference on Computer Vision and Pattern Recognition. 2018, p. 4051- 60.

[31] S Kumar, S Tiwari and SK Singh. Face recognition for cattle. In: Proceedings of $3^{\text {rd }}$ IEEE International Conference on Image Information Processing. 2015, p. 65-72.

[32] T Gaber, A Tharwat, AE Hassanien and V Snasel. Biometric cattle identification approach based on webers local descriptor and AdaBoost classifier. Comp. Electr. Agr. 2016; 122, 55-66.

[33] KP Risha, KA Chempak and CS Sindhu. Difference of gaussian on frame differenced image. Int. J. Innovat. Res. Electr. Electron. Instrum. Contr. Eng. 2016; 3, 92-5.

[34] P Vincent, H Larochelle, I Lajoie, Y Bengio and PA Manzagol. Stacked denoising auto-encoders: Learning useful representations in a deep network with a local denoising criterion. J. Mach. Learn. Res. 2010; 11, 3371-408.

[35] P Vincent, H Larochelle, Y Bengio and PA Manzagol. Extracting and composing robust features with denoising autoencoders. In: Proceedings of the $25^{\text {th }}$ International Conference on Machine Learning. 2008, p. 1096-103.

[36] Y Bengio. Learning deep architectures for AI. Found. Trends Mach. Learn. 2009; 2, 1-127.

[37] Y Bengio, A Courville and P Vincent. Representation learning: A review and new perspectives. IEEE Trans. Pattern Anal. Mach. Intell. 2013; 35, 1798-828. 